\title{
Uso da semiologia na prática da enfermagem: o que sabemos?
}

\author{
Use of semiology in nursing practice: what do we know? \\ Uso de la semiología en la práctica enfermera: ¿qué sabemos?
}

\author{
João Daniel de Souza Menezes \\ ORCID: https://orcid.org/0000-0001-8767-7556 \\ Faculdade de Medicina de São José do Rio Preto, Brasil \\ E-mail: joao.menezes@edu.famerp.br \\ Maria Eduarda Dóro Mota \\ ORCID: https://orcid.org/0000-0002-1977-3578 \\ Faculdade de Medicina de São José do Rio Preto, Brasil \\ E-mail: maria.mota@edu.famerp.br \\ Gabrielle Marques Batista \\ ORCID: https://orcid.org/0000-0002-7475-2593 \\ Faculdade de Medicina de São José do Rio Preto, Brasil \\ E-mail: gabrielle.batista@edu.famerp.br \\ Giovanna Albefaro Sandonato Barbosa \\ ORCID: https://orcid.org/0000-0003-4730-7360 \\ Faculdade de Medicina de São José do Rio Preto, Brasil \\ E-mail: giovanna.barbosa@edu.famerp.br \\ Adriana Pelegrini dos Santos Pereira \\ ORCID: https://orcid.org/0000-0003-4237-365X \\ Faculdade de Medicina de São José do Rio Preto, Brasil \\ E-mail: adrianapelegrini@famerp.br
}

\begin{abstract}
Resumo
Objetivo: Compreender a prática da semiologia na atuação do profissional de enfermagem. Metodologia: Trata-se de um estudo de revisão integrativa da literatura, como critérios de inclusão teve-se artigos publicados entre os anos de 2017 a 2021, nos idiomas português, inglês e espanhol, disponíveis na íntegra e que respondessem à pergunta norteadora "O que vem sendo produzido pelos profissionais em relação ao uso da semiologia pelos profissionais de enfermagem? Foram utilizados os descritores: "Nursing"; "Physical Examination" e "Nursing Process". Resultados: Foram obtidos quatorze artigos, sendo eles a maior parte $(65 \%)$ realizada com base quantitativa, cinco dos artigos selecionados tiveram amostra maior que cinquenta entrevistados. Em relação ao ano de publicação obteve-se um artigo do ano de 2021, quatro de 2020, quatro de 2019, três de 2018 e dois de 2017. Com isso, foram elaborados três subitens para discussão, sendo eles: "Formação profissional em relação ao uso das práticas semiológicas", "Uso da semiologia nas atividades diárias" e "Uso da tecnologia na formação profissional". Assim, é possível compreender que profissional de enfermagem presta assistência em todos níveis de saúde, atendendo desde a rede básica até atendimentos complexos e de reabilitação, em todos esses níveis são necessários atributos esperados do profissional, como avaliação, monitoramento, pensamento crítico, tomada de decisão, entre outros. Conclusão: Em suma, a prática da semiologia permite o cuidado holístico e individualizado, permitindo realizar as atividades diárias de maneira assertiva e precisa.
\end{abstract}

Palavras-chave: Enfermagem; Exame físico; Processo de enfermagem.

\begin{abstract}
Objective: To understand the practice of semiology in nursing professional practice. Methodology: This is an integrative literature review study. The inclusion criteria were articles published between 2017 and 2021, in Portuguese, English and Spanish, available in full and that answered the guiding question "What has been produced by professionals regarding the use of semiology by nursing professionals? The descriptors "Nursing", "Physical Examination" and "Nursing Process" were used. Results: Fourteen articles were obtained, most of them (65\%) with a quantitative basis, five of the selected articles had a sample larger than fifty interviewees. Regarding the year of publication, one article was obtained from the year 2021, four from 2020, four from 2019, three from 2018, and two from 2017. With this, three sub-items were prepared for discussion, being them: "Professional training in relation to the use of semiology practices", "Use of semiology in daily activities" and "Use of technology in professional training". Thus, it is possible to understand that the nursing professional provides care at all levels of health care, from basic care to complex care and rehabilitation, at all these levels, attributes expected of the professional are necessary, such as assessment, monitoring, critical thinking, decision making, among others. Conclusion: In short, the practice of semiology allows holistic and individualized care, allowing performing daily activities assertively and accurately.
\end{abstract}

Keywords: Nursing; Physical examination; Nursing process. 


\begin{abstract}
Resumen
Objetivo: Comprender la práctica de la semiología en la actuación del profesional de la enfermería. Metodología: Se trata de un estudio de revisión integradora de la literatura, como criterio de inclusión se encuentran los artículos publicados entre los años de 2017 a 2021, en los idiomas portugués, inglés y español, disponibles en su totalidad y que responden a la pregunta norteamericana "¿Qué están produciendo los profesionales en relación al uso de la semiología por los profesionales de la enfermería? Se utilizaron los descriptores "Enfermería", "Examen físico" y "Proceso de enfermería". Resultados: Se obtuvieron catorce artículos, la mayoría de ellos (65\%) con base cuantitativa, cinco de los artículos seleccionados tenían una muestra superior a cincuenta entrevistados. En cuanto al año de publicación se obtuvo un artículo del año 2021, cuatro de 2020, cuatro de 2019, tres de 2018 y dos de 2017. Con esto, se prepararon tres subtemas para la discusión, siendo ellos: "Formación profesional en relación con el uso de las prácticas de semiología", "Uso de la semiología en las actividades diarias" y "Uso de la tecnología en la formación profesional". Así, es posible entender que el profesional de enfermería presta asistencia en todos los niveles de salud, atendiendo desde la red básica hasta los cuidados complejos y la rehabilitación, en todos estos niveles se requieren atributos que se esperan del profesional, tales como la evaluación, el monitoreo, el pensamiento crítico, la toma de decisiones, entre otros. Conclusión: En definitiva, la práctica de la semiología permite una atención holística e individualizada, permitiendo realizar las actividades cotidianas de forma asertiva y precisa.
\end{abstract}

Palabras clave: Enfermería; Examen físico; Proceso de enfermería.

\title{
1. Introdução
}

A Enfermagem pode ser compreendida como o cuidado, avaliação e monitoramento das respostas humanas às doenças, para isso torna-se imprescindível o uso de técnicas e conhecimentos para melhor compreensão acerca das possíveis alterações presentes nas respostas às doenças (Geovanini et. al., 2018; Padilha et. al., 2020).

Dessa forma, o uso da semiologia pelos profissionais de saúde é de suma importância para implementação do cuidado, a semiologia pode ser entendida como o estudo dos sinais e sintomas apresentados, para elaborar diagnósticos e, assim, promover o cuidado e reabilitação em sociedade (Sayd et. al., 2021).

Com isso, é notório que com o passar do tempo os profissionais em saúde estão ganhando mais espaço, respaldo e maior conhecimento fazendo com que se tenha maior prestígio e reconhecimento perante a sociedade, o que leva ao aprimoramento de técnicas de conversação e melhores condutas práticas, pois o avanço tecnológico e suas constantes mudanças, faz com que as informações fiquem mais detalhadas e acessíveis a população em geral. Porém, com o avanço tecnológico e científico obteve-se também as subdivisões dos sistemas orgânicos, como cardiológico, pulmonar, gástrico, entre outros, essa divisão acabou levando a focalização da atenção às doenças ao invés do doente, aumentando o uso de exames de imagem, a prática da semiologia perde seu valor diagnóstico em muitas situações cotidianas, na qual os exames complementares se sobressaem a prática clínica (Fonseca et. al., 2009; Chen et. al., 2021; Silva et. al., 2020).

Nos dias atuais a definição do termo saúde auxilia no cuidado humanístico voltado para o paciente como um todo, segundo a OMS (Organização Mundial de Saúde) saúde é um bem estar biopsicossocial e também espiritual, não somente a ausência de doenças, essa definição vem como suporte para o atendimento holístico pelos profissionais em saúde, que em sua maioria focaliza a atenção apenas na doença, esquecendo o contexto de vida do paciente, consequentemente levando a perdas na atenção integral à saúde (Dias et. al., 2020).

Nesse sentido, a fim de estabelecer uma base científica e metodológica, que assegure o profissional de enfermagem tratar as respostas humanas de maneira integral, tem-se o processo de enfermagem, uma ferramenta de trabalho que norteia a atuação profissional, e que ao se dividir em 5 etapas dinâmicas, interligadas e cíclicas, sendo elas: coleta de dados, diagnóstico de enfermagem, planejamento, implementação e avaliação, garantindo assim, um olhar holístico diante das necessidades apresentadas pelo paciente (Souza et. al., 2020 ; Benedet et. al., 2020).

Assim, em relação à categoria coleta de dados, parte que esse trabalho aborda, os profissionais de saúde como um todo necessitam de instrumentos para a coleta das informações subjetivas e objetivas dos pacientes de forma assertiva (Chen et. al., 2021). A anamnese além de ser um fator imprescindível para a criação do vínculo entre o profissional e o cliente, se torna de 
fundamental importância, já que oferece informações como passado, doença pregressa, qualidade de vida, modificações em que o paciente está disposto a enfrentar, oferece subsídio até onde pode-se ir com o tratamento, dando direção na forma do cuidado, já que a assistência deve atender as necessidades individuais, e isso, está relacionado a vivência, cultura, religião e outras características pessoais do cliente, estando relacionada a autopercepção e ao autoconhecimento (Luizari et. al., 2008).

Ainda, os dados objetivos podem ser extraídos através das práticas semiológicas, propedêuticas e pela aferição dos sinais vitais, as formas propedêuticas utilizadas pelos profissionais de saúde se relacionam aos órgãos dos sentidos em sua maior parte, como tato, audição e visão, o profissional utiliza-se da ausculta, percussão, inspeção e palpação, vale ressaltar que em sua maior parte a coleta de dados compreende a inspeção (Benseñor, 2013), essa que pode ser dividida em estática e dinâmica, sendo a observação da estrutura e observação do funcionamento respectivamente. Os dados semiológicos podem ser obtidos por meio da junção entre os achados objetivos e subjetivos, para que dessa forma seja possível compreender toda alteração a nível biológico, psicológico e social que o cliente apresenta (Mattos et. al., 2020).

Dessa forma é possível definir diagnósticos e metas individuais a serem alcançadas para esse paciente, assim, propondo o cuidado de forma assertiva e humanizada. Com isso, esse trabalho tem como objetivo compreender a prática da semiologia na atuação do profissional de enfermagem.

\section{Metodologia}

Trata-se de um estudo de revisão integrativa da literatura, que se utiliza de critérios pré-estabelecidos com a intenção da junção do conhecimento adquirido por meio da leitura e análise criteriosa dos estudos selecionados, e após a síntese desse conhecimento, propor melhorias na prática clínica (Mendes et. al, 2019).

Foram realizadas seis etapas, sendo elas: A- Identificação do tema e seleção da pergunta norteadora; B- Estabelecimento de critérios de inclusão e exclusão dos artigos; C- Definição dos dados a serem obtidos e categorização dos estudos; D- Avaliação dos estudos selecionados; E- Interpretação dos dados obtidos; F- Apresentação dos resultados; (Sousa et. al., 2017).

Como critérios de inclusão teve-se artigos publicados entre os anos de 2017 a 2021, nos idiomas português, inglês e espanhol, disponíveis na íntegra e que respondessem à pergunta norteadora "O que vem sendo produzido pelos profissionais em relação ao uso da semiologia pelos profissionais de enfermagem?". Os critérios de exclusão foram artigos com ano inferior a 2017, não disponíveis na íntegra e que não respondessem à questão norteadora deste estudo.

Com isso, foram utilizadas as bases de dados National Library of Medicine National Institutes of Health (PubMed), biblioteca virtual de saúde (BVS) e Google acadêmico. Foram utilizados os descritores: "Nursing"; "Physical Examination" e "Nursing Process", destaca-se a utilização dos operadores booleanos AND e OR, sendo realizado às buscar no seguinte formato: "Nursing and Physical Examination"; "Physical Examination and Nursing Process" \& "Nursing and Physical Examination or Nursing Process".

Foram encontrados um total de 142.235 artigos, com isso, após os critérios de seleção (período de publicação, idioma e se respondia à pergunta norteadora desse trabalho de revisão) obteve-se um total de 5.378 artigos, dos quais foram efetuado a leitura do título, para compreensão em relação a temática abordada. Após isso, a busca resultou em 328 artigos, que foram selecionados por meio da disponibilidade (artigo disponível na íntegra), com isso, resultou em 95 artigos, sendo efetuada a leitura dos resumos para análise em relação à resposta à pergunta norteadora. Após a leitura dos resumos obteve um total de 14 artigos selecionados para compor essa revisão integrativa da literatura, conforme Imagem 1. 
Imagem 1. Fluxograma de seleção dos artigos.
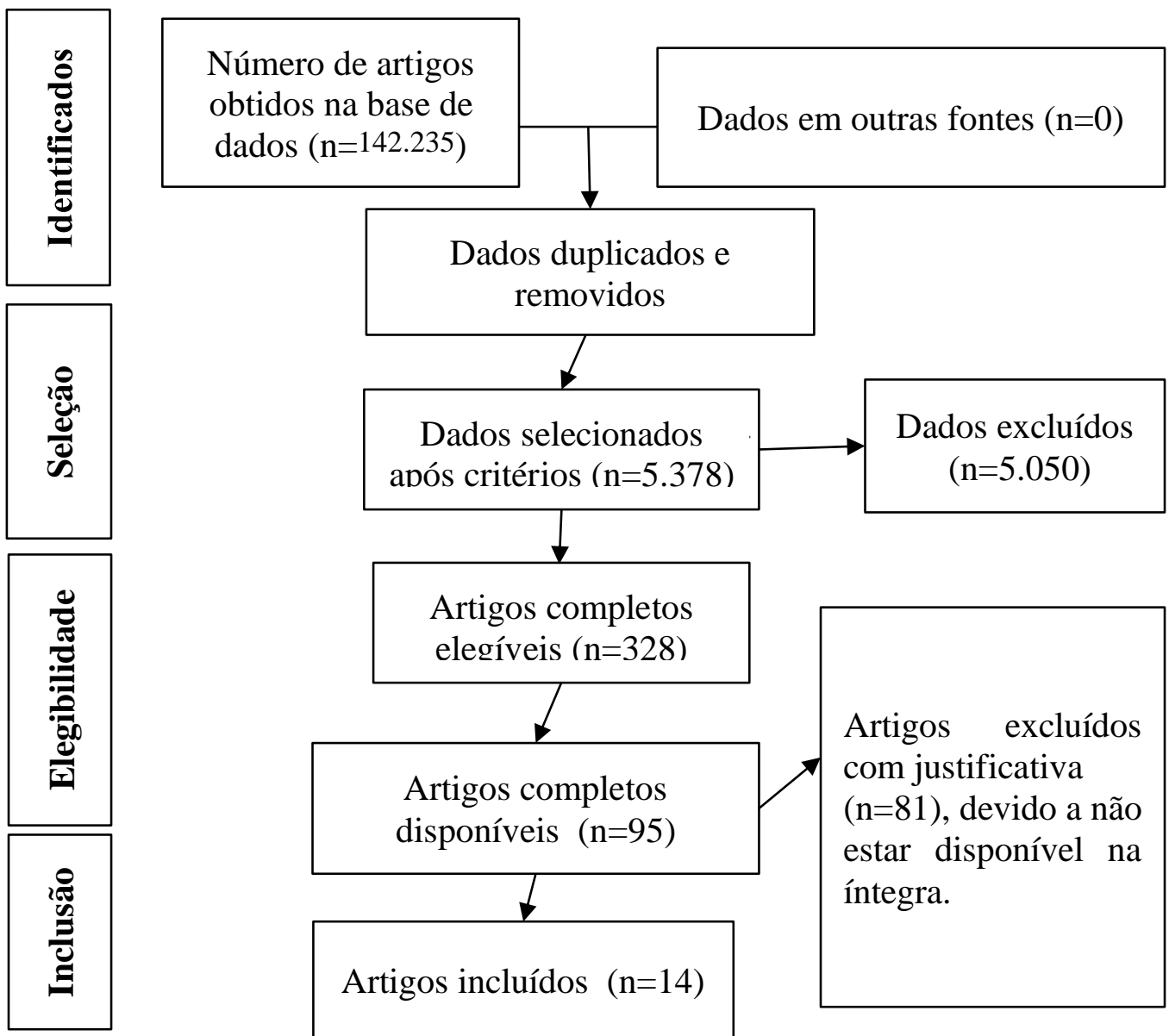

Fonte: Adaptado de Galvão et al (2015).

\section{Resultados e Discussão}

Após o uso dos critérios de inclusão e leitura crítica dos resumos obteve-se um total de quatorze artigos para compor esse estudo. Com isso, para melhor compreensão dos estudos selecionados realizou-se o Quadro 1, conforme autor/ ano, palavrachave, metodologia e amostra.

Dessa forma, em relação aos estudos a maior parte $(65 \%)$ foi realizada com base quantitativa, cinco dos artigos selecionados tiveram amostra maior que cinquenta entrevistados. Em relação ao ano de publicação obteve-se um artigo do ano de 2021, quatro de 2020, quatro de 2019, três de 2018 e dois de 2017. 
Quadro 1. Distribuição dos artigos conforme autor/ ano, palavra-chave, metodologia e amostra. São José do Rio Preto - SP.

\begin{tabular}{|c|c|c|c|}
\hline Autor/ ano & $\begin{array}{l}\text { Palavra chave } \\
\end{array}$ & Metodologia & Amostra \\
\hline Sanongdej et. al., 2021 & $\begin{array}{c}\text { Online course; Information processing } \\
\text { learning theory; Basic physical examination; } \\
\text { Nursing students }\end{array}$ & $\begin{array}{c}\text { Estudo transversal, descritivo e } \\
\text { quantitativo. }\end{array}$ & 202 graduandos \\
\hline Demiray et. al., 2020 & $\begin{array}{l}\text { Nursing; nursing students; physical } \\
\text { examination; simulation }\end{array}$ & $\begin{array}{l}\text { Desenho semi-experimental, } \\
\text { descritivo e quantitativo. }\end{array}$ & 36 graduandos \\
\hline Castro-Yuste et. al., 2020 & $\begin{array}{l}\text { Nursing, Practical; Nursing Faculty Practice; } \\
\text { Health Knowledge, Attitudes, Practice; } \\
\text { Competency-Based Education. }\end{array}$ & Estudo descritivo quantitativo. & 31 entrevistados \\
\hline Fernandes et. al., 2020 & $\begin{array}{l}\text { Enfermagem; Simulação; Exame Físico; } \\
\text { Educação Superior; Aprendizagem. }\end{array}$ & $\begin{array}{l}\text { Estudo quase experimental, } \\
\text { descritivo e quantitativo. }\end{array}$ & 30 graduandos, \\
\hline Melo et. al., 2020 & $\begin{array}{l}\text { Exame Físico; Aplicativos Móveis; } \\
\text { Smartphone; Tecnologia Educacional; } \\
\text { Processo de Enfermagem. }\end{array}$ & Estudo metodológico quantitativo & $\begin{array}{l}9 \text { enfermeiros } \\
\text { docentes }\end{array}$ \\
\hline Alves et. al., 2019 & $\begin{array}{c}\text { Cardiopulmonary Resuscitation; Teaching; } \\
\text { Clinical Competence; Learning; Educational } \\
\text { Measurement. }\end{array}$ & $\begin{array}{c}\text { Estudo de produção tecnológica, } \\
\text { com abordagem quantitativa } \\
\text { descritiva }\end{array}$ & 16 enfermeiros \\
\hline Silva et. al., 2019 & $\begin{array}{c}\text { Avaliação educacional; Aprendizagem; } \\
\text { Educação em Enfermagem; } \\
\text { Metodologia. }\end{array}$ & Estudodescritivo, & 15 docentes \\
\hline Adamy et. al., 2019 & $\begin{array}{c}\text { Enfermagem; Processo de enfermagem; } \\
\text { Engajamento no trabalho; Papel profissional. }\end{array}$ & $\begin{array}{c}\text { Estudo descritivo com abordagem } \\
\text { qualitativa. }\end{array}$ & 12 entrevistados \\
\hline Domingos et. al., 2019 & $\begin{array}{l}\text { Processos de Enfermagem; Validação de } \\
\text { Programas de Computador; Informática em } \\
\text { Enfermagem; Software; Enfermagem. }\end{array}$ & $\begin{array}{l}\text { Estudo descritivo quantitativo, } \\
\text { desenvolvido em três etapas. }\end{array}$ & 100 pacientes \\
\hline Gadioli et. al., 2018 & $\begin{array}{l}\text { Ensino; Enfermagem; Tecnologia } \\
\text { Educacional; Exame físico; Ensino de } \\
\text { Enfermagem e Semiologia. }\end{array}$ & $\begin{array}{l}\text { Trata-se de um estudo } \\
\text { metodológico quantitativo }\end{array}$ & $\begin{array}{l}3 \text { peritos especialistas } \\
\text { na área da } \\
\text { enfermagem, } 3 \\
\text { informática e com } 20 \\
\text { graduandos de } \\
\text { enfermagem. }\end{array}$ \\
\hline Moser et. al., 2018 & $\begin{array}{l}\text { UTI; Enfermagem; Sistematização da } \\
\text { Assistência de Enfermagem. }\end{array}$ & $\begin{array}{c}\text { Trata-se de um estudo qualitativo } \\
\text { descritivo-exploratório. }\end{array}$ & 4 enfermeiros. \\
\hline Macedo et. al., 2018 & $\begin{array}{c}\text { Enfermagem; Ensino; Estágios; Exame Físico; } \\
\text { Comportamento. }\end{array}$ & Pesquisa descritiva qualitativa & 62 graduandos \\
\hline Boaventura et. al., 2017 & $\begin{array}{c}\text { Enfermagem; Educação; Processos de } \\
\text { Enfermagem }\end{array}$ & $\begin{array}{l}\text { estudo exploratório-descritivo, de } \\
\text { abordagem quali-quantitativa. }\end{array}$ & 164 enfermeiros. \\
\hline Melo et. al., 2017 & $\begin{array}{c}\text { Educação em Enfermagem; Estudantes de } \\
\text { Enfermagem; Conhecimento; Cuidados de } \\
\text { Enfermagem; Pesquisa em Avaliação de } \\
\text { Enfermagem. }\end{array}$ & $\begin{array}{l}\text { Estudo quantitativo, descritivo e } \\
\text { analítico }\end{array}$ & 186 graduandos \\
\hline
\end{tabular}

Fonte: Autores.

O profissional de enfermagem presta assistência em todos níveis de saúde, atendendo desde a rede básica até atendimentos complexos e de reabilitação, em todos esses níveis são necessários atributos esperados do profissional, como avaliação, monitoramento, pensamento crítico, tomada de decisão, entre outros, estes atributos são melhor trabalhados com uso do processo de enfermagem, ferramenta de trabalho do profissional, que oferece suporte, segurança e respaldo legal nas decisões clínicas perante a assistência ao paciente (Melo et. al., 2017 ; Gadioli et. al., 2018).

Assim, para o profissional realizar o PE de forma assertiva é necessário embasamento clínico, por meio dos dados obtidos na etapa coleta de dados ou primeira etapa do processo (Macedo et. al., 2018). A realização de um diagnóstico adequado, que atende as necessidades do cliente, depende de uma avaliação coerente, o que impõe técnicas acuradas para obtenção de dados, com a realização de propedêuticas de maneira adequada e avaliação precisa dos dados obtidos (Domingos et. al., 2019 ; Melo et. al., 2020).

Ademais, é por meio do uso da semiologia que o profissional obtém grande parte dos dados da primeira etapa do PE, 
necessários para a elaboração do diagnóstico e metas de cuidado para com o paciente.

Dessa forma, foram elencadas três subcategorias para discussão, sendo elas: "Formação profissional em relação ao uso das práticas semiológicas", "Uso da semiologia nas atividades diárias" e "Uso da tecnologia na formação profissional".

\section{Formação profissional em relação ao uso das práticas semiológicas}

O primeiro contato entre o graduando e o seu paciente pode ser vivenciado de diversas formas, uma delas é com o receio em abordar seu cliente ou em como realizar a anamnese e obter as informações mais relevantes sem ser indelicado e invasivo, esse primeiro contato é fundamental no desenvolvimento profissional e deve estar embasado com conhecimento adquiridos anteriormente em relação às formas de comunicação verbal e não verbal, formas de apresentação, atributos da segurança do paciente, como identificação correta, grades e cabeceiras elevadas, higiene das mãos e conhecimento prévio sobre microbiologia. Além disso, é indispensável o conhecimento sobre as propedêuticas utilizadas no exame físico e anamnese geral, obtendo dados de moradia, transporte, segurança, trabalho e situação econômica, história pregressa e correta interpretação/ junção destes dados com a avaliação física (Macedo et. al., 2018).

Com isso, em relação a realização da anamnese, que pode ser vivenciada na graduação com estranheza, pois algumas perguntas ainda possuem tabus sociais, como a abordagem da sexualidade, uso de drogas, abortos pregressos, prostituição, doenças sexualmente transmissíveis, entre outros assuntos que podem gerar dificuldade em como questionar o paciente, pela reação de invasão que pode ser gerada. Para isso, durante a graduação, assuntos com a possibilidade de gerar estranheza devem ser instigados e discutidos, para facilitar a prática clínica futura, já que assuntos não abordados de forma assertiva e segura podem mudar consideravelmente o desfecho no cuidado (Melo et. al., 2017).

Além da anamnese, para a correta realização das propedêuticas, necessita-se de constante educação e atualização na prática e interpretação dos dados obtidos, para que assim, se tenha habilidade, atitude e conhecimento não só na realização, mas na tomada de decisão e prosseguimento perante os dados obtidos, uma vez que os diagnósticos de enfermagem dependente fundamentalmente dos dados objetivos e subjetivos (Adamy et. al., 2019).

Nesse sentido, é evidente que o profissional enfermeiro deve fazer uso da semiologia e das relações interpessoais como mecanismo de busca de dados que sejam interessantes para os cuidados de enfermagem, para isso, as matérias consideradas básicas para os cursos em saúde devem estar inseridas e compreendidas em sua totalidade. Dessa forma, cabe ao professor ser o facilitador no processo de aprendizagem, criando condições para produzir reflexão e pensamento crítico no aluno, o que faz em junção aos conhecimentos prévios básicos adquiridos o sentimento de motivação, muitas vezes relacionados ao sentir-se ativo, importante e com autonomia (Fernandes et, al., 2020).

Assim, a realização da coleta da história clínica, bem como a consulta de enfermagem deve ser atributo fundamental do profissional de enfermagem, o que faz com que este alcance o respaldo e segurança na sua atuação, alcançando assim o reconhecimento profissional, parte tão desejada (Adamy et. al., 2019).

Com isso, é notório que a formação do profissional de enfermagem depende fundamentalmente de prática, já que o cuidado humanizado depende de recursos humanos com conhecimento e habilidades prévias, para assim, tomar as escolhas terapêuticas fundamentadas. $\mathrm{O}$ acadêmico de enfermagem em muitos casos não recebe formação estruturada na prática clínica, dificultando a atuação profissional, para isso, é fundamental a realização de estágios em contato direto com o paciente e população, com auxílio de professores nas dificuldades e lacunas encontradas pelo estudante.

Dessa forma, as instituições formadoras necessitam de reestruturação no ensino da semiologia, para que se tenha carga horária compatível com o conteúdo e práticas adequadas, com contato constante com as propedêuticas, levando ao desenvolvimento da habilidade na realização (Demiray et. al., 2020). 


\section{Uso da semiologia nas atividades diárias}

O enfermeiro em atuação diária, utiliza-se do processo de enfermagem, atributo de suma importância, uma vez que oferece respaldo e coordena o trabalho profissional (Macedo et. al., 2018). O PE é composto por cinco etapas como já mencionado, etapas cíclicas e interdependentes, o primeiro passo para a realização do processo é a coleta dos dados, nessa parte o profissional buscar identificar possíveis alterações nas respostas humanas, compreende os dados objetivos como os sinais vitais e respostas obtidas através da realização das propedêuticas, já os dados subjetivos são os dados que não conseguimos obter através do exame físico, mas sim pelo relato do paciente, como história pregressa, relato de dor, mal estar, medo, ansiedade, dados referente aos familiares, trabalho, estudo, moradia e outros, esta parte é conhecida como anamnese, palavra derivada do grego, com sentido de buscar-se algo ou trazer recordações, essas duas partes, dados objetivos e subjetivos devem ser correlacionados para a formação da coleta de dados ( Melo et. al., 2020).

Com isso, ainda em relação a etapa coleta de dados, é através da sua realização que se obtém o vínculo entre o profissional e o cliente, vínculo este que pode ser positivo ou negativo, dependendo da forma de abordagem. É necessário questionar o paciente de forma harmônica, não invasiva, fluída e que ofereça sensação de segurança. Ainda nessa etapa são definidos os critérios de direção no cuidado, com os limites de perdas e ganhos que o paciente está disposto a enfrentar para o seu tratamento, além da elaboração de estratégias para prosseguir o cuidado, já que o paciente deve estar incluso na tomada de decisão no que refere ao seu cuidado (Moser et. al., 2018).

Além disso, na etapa seguinte, diagnóstico de enfermagem, parte está em que o profissional irá elencar alterações passíveis de mudança, na qual o seu cuidado dependerá das decisões tomadas, atualmente há algumas taxonomias de diagnósticos de enfermagem, a mais conhecida é a NANDA-I, um padrão norte-americano de uso mundial. O uso de taxonomias é feito para que todos os profissionais se comuniquem da mesma forma, evitando assim entendimentos equivocados de uma mesma situação (Domingos et. al., 2019 ; Melo et. al., 2020).

Após isso, é realizado o planejamento de enfermagem, com a elaboração de metas a serem alcançadas, assim, se faz próxima etapa, a implementação por toda equipe de enfermagem, sendo estes o enfermeiro(a), técnico e auxiliar de enfermagem, após a implementação dos cuidados, é realizado a avaliação de enfermagem para compreender se a meta foi alcançada e quais alterações serão necessárias para a conclusão da meta estabelecida (Boaventura et. al., 2017).

Dessa forma, a prática da semiologia e semiotécnica é de fundamental importância para a atuação do profissional e vem como auxílio na interpretação das respostas humanas, possibilitando a avaliação e monitoramento das respostas às doenças, o uso da semiologia está vinculada ao cuidado, sendo de grande importância o uso de forma coerente e segura, o que além de obter dados, possibilita confiança do paciente no cuidado oferecido. O uso da semiologia deve ser instigado desde a graduação, pois a prática semiológica oferece respaldo nas ações e diagnósticos de enfermagem adequados, possibilitando a monitorização e avaliação diária do paciente, além de transmitir segurança e eficiência (Fernandes et. al., 2020).

Em suma, o uso das propedêuticas permite a avaliação contínua da assistência oferecida, bem como as respostas ao tratamento de toda equipe de saúde, médicos, fisioterapêuticas, fonoaudiólogos e outros, uma vez que o profissional de enfermagem muitas vezes é o responsável pela interface entre o paciente e demais profissionais em saúde, o que faz com que as terapêuticas propostas sejam avaliadas em continuidade, obtendo assim, mudanças significativas no desfecho clínico (Moser et. al., 2018 ; Adamy et. al., 2019).

\section{Uso da tecnologia na formação profissional}

Com a evolução tecnológica e os recursos de mídias disponíveis, o processo de aprendizagem se torna eficiente e dinâmico, o uso de manequins automatizados com recursos de expressão e respostas controladas faz o aprendizado didático e 
intuitivo, o que colabora para a formação de profissionais de excelência (Fernandes et .al., 2020 ; Silva et. al., 2019).

Atualmente, se tem diversos cenários em que esses manequins estão disponíveis para a formação complementar nos diversos cursos em saúde, como enfermagem e medicina, os quais utilizam desse método de aprendizagem desde o início da graduação, possibilitando aprimoramento de técnicas de conversação e abordagem do paciente, prática da semiologia, como ausculta cardíaca, pulmonar e abdominal, tanto fisiológicas como patológicas. Ainda, há manequins com tecnologias superiores que reproduzem parada cardiorrespiratória, parto, situações de urgência e emergência como choque anafilático, elevando a prática da habilidade, raciocínio crítico e atitude profissional, formando profissionais de excelência para o mercado de trabalho (Macedo et. al., 2019; Gadioli et. al., 2018).

Assim, o uso desses recursos produz aprendizado eficiente, quando o graduando entra em contato com situações que fogem da rotina do dia a dia, mas em ambiente monitorado e com suporte, faz com que esse estudante se torne diferenciado dos demais, melhorando sua habilidade em situações inesperadas futuras, já que houve adequado compreendimento dos diversos tipos de cenários em que sua atuação será fator imprescindível para a adequada condução da situação (Fernandes et. al., 2020 ; Demiray et. al., 2020).

Com isso, algumas técnicas estão sendo empregadas na formação, como o recurso OSCE (Objective Structured Clinical Examination), ferramenta baseada nos princípios da objetividade e padronização de técnicas de avaliação do estudante em cenários de atuação clínica simuladas, na qual o estudante deve resolver situações estabelecidas, desenvolvendo raciocínio clínico e tomada de decisão. Além disso, há rotatividade nas estações de simulação ou cenário, fazendo com que se tenha diversas perspectivas a serem abordadas no estudante, o que facilita o desenvolvimento de habilidades, atitudes e do conhecimento sobre determinado assunto (Alves et. al., 2019).

Dessa forma, o uso da ferramenta OSCE a princípio desenvolvida para os estudantes de medicina, tem ganhado espaço na formação dos profissionais enfermeiros, para isso, necessitou de alterações no seu formato original idealizado, passando a atender de maneira assertiva os graduandos do curso de enfermagem, produzindo adequadamente as respostas e atitudes esperadas pelo mercado de trabalho (Castro-Yuste et. al., 2020; Gadioli et. al., 2018).

Ainda, o recurso OSCE permite avaliar de forma objetiva determinadas competências, possibilitando a implementação do raciocínio crítico no qual os profissionais de enfermagem estão inseridos, isso durante o período de graduação, o que possibilita o remodelamento dos estudantes, capacitando-os de forma segura e de excelência. Por meio do OSCE é possível desenvolver no acadêmico atributos básicos, como aferição e avaliação dos sinais vitais, exame físico, segurança do paciente em relação aos cuidados básicos como a administração de medicamentos, identificação correta, higiene das mãos, não transmissão cruzada de patógenos, além de permear situações adversas como queda, comunicação de morte ao familiar, erro de medicação e outras situações adversas, possibilitando abordar todo processo de enfermagem (Castro-Yuste et. al., 2020 ; Sanongdej et. al., 2021).

\section{Conclusão}

Dessa forma, pode-se compreender que a semiologia está envolta em todo cuidado de enfermagem, permeando desde atenção básica até assistência em reabilitação em saúde. $\mathrm{O}$ uso da semiologia permite identificar de forma precoce alterações nas respostas humanas, também oferece preditivos na monitorização do estado de saúde e das respostas às doenças. Ainda, é necessário maior atuação e compreensão em relação ao uso do processo de enfermagem e a utilização das propedêuticas como forma de respaldo nas atividades diárias do profissional enfermeiro, isso desde o período de graduação.

Em suma, a prática da semiologia permite o cuidado holístico e individualizado, permitindo realizar as atividades diárias de maneira assertiva e precisa. Assim, como sugestão para trabalhos futuros, tem-se a realização de trabalhos em campo sobre 
como a prática do processo de enfermagem interfere no reconhecimento profissional, por meio da perspectiva do enfermeiro e estudos em relação às lacunas vivenciadas durante a graduação.

\section{Referências}

Adamy, E. K, Zocche, D. A. D. A. \& Almeida, M. D. A. (2019). Contribuição do processo de enfermagem para a construção identitária dos profissionais de enfermagem. Revista Gaúcha de Enfermagem, 41.

Alves, M. G., Carvalho, M. T. M., Nascimento, J. D. S. G., Oliveira, J. L. G. D., Cyrillo, R. M. Z., Braga, F. T. M. M., \& Dalri, M. C. B. (2019). Construção e validação de Exame Clínico Objetivo Estruturado (OSCE) sobre ressuscitação cardiopulmonar. Revista Mineira de Enfermagem, $23,1-9$.

Benedet, S. A., Padilha, M. I., Peres, M. A. D. A. \& Bellaguarda, M. L. D. R. (2020). Características essenciais de uma profissão: análise histórica com foco no processo de enfermagem. Revista da Escola de Enfermagem da USP, 54.

Benseñor, I. M. (2013) Anamnese, exame clínico e exames complementares como testes diagnósticos / Clinical examination and laboratory investigation as diagnostic tests. Rev Med (São Paulo), 00(4):236-41.

Boaventura, A. P, Santos, P. A. \& Duran, E. C. M. (2017). Conhecimento teórico e prático do enfermeiro sobre o Processo de Enfermagem e a Sistematização da Enfermagem. Enferm Glob, 16 (2), 182-216.

Castro-Yuste, C., Rodríguez-Cornejo, M. J, García-Cabanillas, M. J. G. C, de Carmen Paublete-Herrera, M., Paramio-Cuevas, J. C. \& Moreno-Corral, L. J. (2020). Projeto de um exame clínico estruturado objetivo de enfermagem de um programa de prática clínica do primeiro ano. Revista da Escola de Enfermagem da USP, 54.

Chen, S. H, Chen, S. C, Lai, Y. P, Chen, P. H. \& Yeh, K. Y. (2021). O objetivo estruturou o exame clínico como uma estratégia de avaliação da competência clínica em profissionais de enfermagem iniciantes em Taiwan. Enfermagem BMC, 20 (1), 1-9.

da Silva, J. C. B., da Silva, L. C., Lemos, M. E. P., Mota, S. M. A. \& de Paula, W. K. A. S. (2019). A percepção dos docentes sobre o exame clínico objetivamente estruturado no ensino-aprendizagem de enfermagem. Pesquisa em foco, 24(2).

de Sousa, L. M. M., Marques-Vieira, C. M. A., Severino, S. S. P. \& Antunes, A. V. (2017). A metodologia de revisão integrativa da literatura em enfermagem. No21 Série 2-Novembro 2017, 17.

de Souza, G. B., da Silva Santiago, A. X., dos Santos, O. P., Pereira, B. A., de Souza Caetano, S. R. \& dos Santos, C. C. (2020). Sistematização da assistência de enfermagem e processo de enfermagem: conhecimento de graduandos. Brazilian Journal of Health Review, 3(1), $1250-1271$.

Demiray, A., Kiziltepe, S. K, İlaslan, N. \& Açil, A. (2020). O Efeito da Simulação de Alta Fidelidade na Melhoria das Habilidades de Exame Físico de Estudantes de Enfermagem. International Journal of Nursing , 7 (1), 8-17.

Dias, F. A., Pereira, E. R. \& Silva, R. M. C. R. A. (2020). Espiritualidade e saúde: uma reflexão crítica sobre a vida simbólica. Research, Society and Development, 9(5), e52953113-e52953113.

Domingos, C. S, Boscarol, G. T, Souza, C. C. D, Tannure, M. C, Chianca, T. C. M. \& Salgado, P. D. O. (2019). Adaptação de software com o processo de enfermagem para unidades de internação. Revista Brasileira de Enfermagem, 72 , 400-407.

Fernandes, R. M, Carino, A. C. C, Fernandes, M. I. D. C. D, Tinôco, J. D. D. S, Ribeiro, H. C. T. C. \& Lira, A. L. B. D. C. (2020). Ensino do exame físico cardiovascular em enfermagem: simulação clínica. Revista Brasileira de Enfermagem , 73.

Fonseca, L. M. M., Góes, F. D. S. N. D., Ferecini, G. M., Leite, A. M., Mello, D. F. D. \& Scochi, C. G. S. (2009). Inovação tecnológica no ensino da semiotécnica e semiologia em enfermagem neonatal: do desenvolvimento à utilização de um software educacional. Texto \& Contexto-Enfermagem, 18, 549-558.

Galvão, T. F., Pansani, T. D. S. A. \& Harrad, D. (2015). Principais itens para relatar Revisões sistemáticas e Meta-análises: A recomendação PRISMA. Epidemiologia e Serviços de Saúde, 24, 335-342.

Gadioli, B., Fulquini, F. L, Kusumota, L., Gimenes, F. R. E. \& Carvalho, E. C. D. (2018). Construção e validação de um objeto virtual de aprendizagem para o ensino da semiologia vascular venosa periférica. Escola Anna Nery, 22 anos.

Geovanini, T., Moreira, A., Dornelles, S. \& Machado, W. C. A. (2018). História da Enfermagem: versões e interpretações. Thieme Revinter Publicações LTDA.

Macedo, Í. L. J. D, Zamarioli, C. M. \& Carvalho, E. C. D. (2018). Incidentes envolvendo envolvendo Semiologia nas atividades práticas de graduandos de enfermagem. Revista Brasileira de Enfermagem, 71 , 1572-1579.

Mattos, W., Hilbig, A., Tovo, C. V., de Souza Meyer, E. L., de Lima, M. R. A. A. \& da Silva, N. B. (2020). Semiologia do Adulto: Diagnóstico Clínico Baseado em Evidências. Medbook.

Melo, E. B. M. D., Primo, C. C., Romero, W. G., Sant'Anna, H. C., Sequeira, C. A. D. C, Lima, E. D. F. A. \& Fioresi, M. (2020). Construção e validação de aplicativo móvel para o desenvolvimento de histórico e diagnóstico de enfermagem. Revista Brasileira de Enfermagem, 73.

Melo, G. D. S. M., Tibúrcio, M. P., Freitas, C. C. S. D., Vasconcelos, Q. L. D. D. A. Q. D., Costa, I. K. F. \& Torres, G. D. V. (2017). Semiologia e semiotécnica da enfermagem: avaliação dos conhecimentos de graduandos sobre procedimentos. Revista Brasileira de Enfermagem , 70 , $249-256$. 
Research, Society and Development, v. 10, n. 12, e529101220703, 2021 (CC BY 4.0) | ISSN 2525-3409 | DOI: htttp://dx.doi.org/10.33448/rsd-v10i12.20703

Mendes, K. D. S., Silveira, R. C. D. C. P. \& Galvão, C. M. (2019). Uso de gerenciador de referências bibliográficas na seleção dos estudos primários em revisão integrativa. Texto \& Contexto-Enfermagem, 28.

Moser, D. C., Aguiar da Silva, G., Rodrigues de Oliveira Maier, S., Costa Barbosa, L. \& Gaffuri da Silva, T. (2018). Sistematização da assistência de enfermagem: a percepção dos enfermeiros. Revista de Pesquisa: Cuidado e Fundamental , 10 (4).

Sanongdej, W., Leelacharas, S., Partiprajak, S. \& Kittipimpanon, K. (2021). Investigating Learning Process Factors Relacionados à Aprendizagem Online e Exames Físicos entre Estudantes de Enfermagem.

Sayd, J. D., Silva, D. D. A. \& Ribeiro, M. P. D. (2021). O aprendizado de semiologia em um currículo tradicional. Revista Brasileira de Educação Médica, 27, 104-113.

Padilha, M. I., Kuhnen, A. E., Ferreira, A. C., Maia, A. R. C. R., Koerich, A. M. E., de Almeida Filho, A. J.,... \& dos Santos, I. (2020). Enfermagem: história de uma profissão. Difusão Editora.

Silva, R. P., Camacho, A. C. L. F., da Silva, M. A. P. \& de Menezes, H. F. (2020). Estratégias do uso de metodologia ativa na formação de acadêmicos de enfermagem: relato de experiência. Research, Society and Development, 9(6), e160963543-e160963543. 